\title{
Efficiency of Epidurally Injected lidocaine, lidocaine and Morphine or Lidocaine and Tramadol for Postoperative Analgesia in Dogs Following Orchiectomy and Ovariohysterectomy
}

\author{
Alan Maksimović* and Ismar Lutvikadić \\ Department of Veterinary Surgery, University of Sarajevo - Veterinary Faculty, Bosnia and Herzegovina \\ *Corresponding author: alan.maksimovic@vfs.unsa.ba
}

\begin{abstract}
\begin{tabular}{llll}
\hline Article History: 20-009 Received: 12-Jan-20 & Revised: 05-Aug-20 & Accepted: 18-Aug-20
\end{tabular}
\section{ABSTRACT}

The objective of this study was to evaluate and compare the postoperative analgesia provided by epidural lidocaine, lidocaine and morphine or lidocaine and tramadol in dogs following elective orchiectomy or ovariohysterectomy. Thirty-six clinically healthy dogs, classified as class I and II according to the American Society of Anesthesiologists classification system, were randomly allocated into three groups of 12 each, with six females and six males. The dogs received $4 \mathrm{mg} / \mathrm{kg}$ of lidocaine combined with $0.01 \mathrm{ml} / \mathrm{kg}$ of $0.9 \% \mathrm{NaCl}, 0.1 \mathrm{mg} / \mathrm{kg}$ of morphine or $1.0 \mathrm{mg} / \mathrm{kg}$ of tramadol epidurally. Postoperative pain was assessed 4, 8, 12, 18 and 24 hours after epidural anaesthesia using the University of Melbourne pain scale (UMPS), by a single male observer who was blinded to the epidural protocol. According to the obtained results, evaluated epidural protocols provided sufficient and comparable postoperative analgesia throughout the complete 24-hours observation period. The appearance of vocalization or painful reaction to palpation of the wound surrounding tissue were absolutely absent. Values of 10 points, or higher, indicating the presence of moderate to severe pain and the necessity of additional analgesia, were not recorded. However, established quality of postoperative analgesia is probably not the sole result of epidural administration of drugs, but also analgesic activity of drugs used for general anaesthesia (xylazine and ketamine), and their mutual synergistic or additive effects.
\end{abstract}

Key words: Dog, Epidural anaesthesia, Postoperative pain.

(C2020 IJVS - All Rights Reserved

\section{INTRODUCTION}

Epidural anaesthesia (EA) is a method of drug administration in close proximity to their site of action. This way binding for specific receptors is maximized, resulting in more potent analgesia with reduction of total dose comparing to the used drug systemic administration (Torske and Dyson 2000). In addition, EA reduces stress response to surgery through prevention of the endocrine and metabolic response to trauma (Desborough 2000); and significantly reduces the dose of injection and volatile general anaesthetics (Hodgson et al. 1999; Rauser et al. 2004; Tusell et al. 2005; Almeida et al. 2007; Qayyum et al. 2020). In dogs, it is advocated for different surgical procedures caudal to the diaphragm (Wetmore and Glowaski, 2000; Valverde, 2008). The most commonly used epidural anaesthetic is lidocaine (Habibian et al. 2011; Gusak et al. 2013; Mwangi 2020). The addition of opioids to epidural local anaesthetics provide more potent and prolonged analgesia (Valverde, 2008). Epidural application of morphine provides much longer duration of its analgesic effect over its administration by any other routes (Almeida et al. 2010). Tramadol, a synthetic racemic mixture of the 4-phenyl-piperidine analogue of codeine, has received widespread acceptance in human medicine since it was first introduced in 1977 in Germany (Habibian et al. 2011). Interest for epidural use of tramadol in veterinary analgesia emerged over the past decade, with relatively few studies conducted in dogs (Halder and Bose, 2000; Guedes et al. 2005; Vettorato et al. 2010; Almeida et al. 2010; Mastrocinque et al. 2012; Neves et al. 2012). It has been shown that epidural tramadol has analgesic effect comparable to that of epidural morphine (Almeida et al. 2010; Neves et al. 2012). Discovered synergistic relationship between local anaesthetic and opioids with resulting more potent, prolonged analgesia and faster onset of action (Wetmore and Glowaski, 2000; Almeida et al. 2010; Habibian et al. 2011), proved to be excellent for ensuring adequate pre, intra and postoperative analgesia (Hendrix et al. 1996;

Cite This Article as: Maksimović A and Lutvikadić I, 2021. Efficiency of epidurally injected lidocaine, lidocaine and morphine or lidocaine and tramadol for postoperative analgesia in dogs following orchiectomy and ovariohysterectomy. International Journal of Veterinary Science 10(1): 13-18. https://doi.org/10.47278/journal.ijvs/2020.015 
Amnikov and Zlateva, 2004). Almeida et al. (2010) provided comparison of postoperative analgesic efficiency of pre-emptive epidural administration of lidocaine alone, or in combination with morphine or tramadol, following orchiectomy in dogs. To the best of our knowledge, similar research was not conducted for surgical procedure of ovariohysterectomy. Generally, there are few studies which investigated analgesic efficiency of different epidural protocols for surgical procedure of ovariohysterectomy in dogs (Amnikov and Zlateva 2004; Acosta et al. 2005; Almeida et al. 2007; Pekcan and Koc 2010; Pohl et al. 2012; Neves et al. 2012). The aim of this study was to assess postoperative analgesic efficiency of pre-emptive epidural administration of lidocaine alone, or in combination with morphine or tramadol, in dogs submitted to orchiectomy or ovariohysterectomy; and to compare obtained pain scores between these two surgical procedures.

\section{MATERIALS AND METHODS}

This work was approved by the Ethic Committee of the Veterinary Faculty University of Sarajevo, and the owner's signed written voluntary consent was obtained for each animal.

\begin{abstract}
Animals
The study was performed in 36 healthy (according to the American Society of Anesthesiologists classification system ASA, class I and II) mixed breed dogs, 18 males and 18 females, allocated into three groups of 12, marked according to epidural protocols as group L (lidocaine), group LT (lidocaine + tramadol) and group LM (lidocaine + morphine). The dogs were 19.6 \pm 11.3 (mean \pm SD) months old $(18.4 \pm 12.3,20.5 \pm 8.7$ and $19.8 \pm 13.4$ months in the L, LT and LM groups, respectively), weighing $16.5 \pm 5.7 \mathrm{~kg}(13.5 \pm 3.7,15.9 \pm 3.8$ and $20.0 \pm 7.2 \mathrm{~kg}$ in the $\mathrm{L}$, LT and LM groups, respectively). Physical examination and routine blood testing of all dogs were performed to estimate their health status and exclude the presence of contraindication for the EA. Potential errors of postoperative pain evaluation were minimized by excluding unfriendly, overfriendly, frightened, pregnant, lactating and animals in estrus and those that had received analgesics within 15 days of surgery.
\end{abstract}

\section{Anaesthesia and Surgeries}

In all of the dogs the food was withheld minimally 8 hours prior to induction of general anaesthesia, with unrestricted access to water. The animals were randomly allocated, by drawing paper numbers papers from two envelopes (one with female and other with male dogs), into three groups of six males and six females in each. All dogs received epidurally $4 \mathrm{mg} / \mathrm{kg}$ of lidocaine (lidokain hidrohlorid 2\%, Galenika, Serbia) combined with $0.01 \mathrm{ml} / \mathrm{kg}$ of $0.9 \% \mathrm{NaCl}$ in group $\mathrm{L}, 0.1 \mathrm{mg} / \mathrm{kg}$ of morphine (morphini hydrocloridum $20 \mathrm{mg} / \mathrm{ml}$, Alkalod, Macedonia) in group LM or $1.0 \mathrm{mg} / \mathrm{kg}$ of tramadol (TramadolSTADA $100 \mathrm{mg} / 2 \mathrm{ml}$ STADA - Arzneimittel AG, Germany) in group LT. Induction of general anaesthesia and immobilization of the animals for epidural injections was achieved with intravenous bolus mixture $5 \mathrm{mg} / \mathrm{kg}$ of ketamine hydrochloride (Ketaminol ${ }^{\mathrm{TM}} 10$,
Intervet International B.V., Netherland) and $1 \mathrm{mg} / \mathrm{kg}$ of xylazine (Xylazine Bio 2\%, Bioveta, Czech Republic). The trachea was intubated and animals positioned in sternal recumbency. The EA was performed as described by Valverde (2008), in sternal recumbency of the animals, by the same person, after area of lumbosacral space was clipped and aseptically prepared. Correct positioning of the needle was identified by the absence of cerebrospinal fluid or blood at the hub, hanging drop technique and lack of resistance to drug injection (Torske and Dyson 2000; Jones 2001; Velverde 2008). The efficiency of EA was confirmed by observation of anal sphincter relaxation and loss of pedal withdrawal reflex tested by mild clamping stimulus on the hind limbs. Sternal positioning of the animals was maintained for 20 minutes after EA to provide uniform distribution of the injected drugs (Almeida et al. 2010). Surgical plane of anaesthesia was maintained subsequently with intravenous ketamine and xylazine to effect. Depth of anaesthesia was subjectively evaluated by assessment of the muscular tonus of the jaw, palpebral reflex, dilatation of the pupils, position of the eyeballs and response to surgery. During surgery, heart rate, respiratory rate, arterial hemoglobin oxygen saturation and arterial blood pressure (non-invasive oscillometric) were monitored (PM-9000Vet, Mindray, China). The surgeries were performed by the same surgeon and did not last longer than 20 minutes. Sterile saline solution was applied at a rate of $10 \mathrm{ml} / \mathrm{kg} /$ hour to all animals over intravenous cephalic catheter during the procedure.

\section{Postoperative Pain Assessment}

All dogs were housed for 24 hours pre-operative in adequate individual accommodation to allow partial adaptation to the specific clinical environment, and therefore, to minimize influence of the environmental stressors on the behaviour of the dogs during postoperative analgesia evaluation. For the same reason, during the entire preoperative period the interaction with the dogs was performed by the same person, who also evaluated quality of postoperative analgesia (Moll et al. 2011). During preoperative 24 hours period of adaptation, determination of basal mental status was performed according to Firth and Haldane (1999), same as values of the pulse and respiration frequency and body temperature.

Postoperative analgesia was assessed 4, 8, 12, 18 and 24 hours after epidural anaesthesia using the University of Melbourne pain scale UMPS, by a single male observer who was blinded to the epidural protocol. Within the aforementioned evaluation system, the value of 0 point represented the complete absence of behavioural and physiological indicators of pain, while the maximum value of 27 points indicated presence of the most intense pain possible. Adequate postoperative analgesia was considered to be up to value of 10 points, and intramuscular morphine $(0.2 \mathrm{mg} / \mathrm{kg})$ was reserved if the evaluation score exceeds this value (Almeida et al. 2010) Activity, position, vocalization and breathing frequency were evaluated by observation, prior to the interaction with the dog, in order to reduce stress interference. For the same reason, the assessment of the physiological data was performed minimally 5 minutes after evaluator entered the area of postoperative hospitalization. Breathing frequency 
was established by observing the motion of the chest, the cardiac rhythm by auscultation and palpation of the peripheral pulse and the body temperature by rectal thermometry. The final procedure in the assessment of postoperative analgesia was evaluation of the reaction to palpation of the area of the surgical wound (Moll et al. 2011). According to the recommendations (Holton et al. 2001; Morton et al. 2005) obtained values of physiological and behavioural parameters were also independently evaluated.

\section{Statistical Analysis}

Duration of the surgical procedure and the score of the postoperative pain assessment were compared between the observed groups of animals for the presence of statistically significant differences using a one-way ANOVA test. Values of the postoperative pain evaluation were also compared between females and males (post ovariohysterectomy and orchiectomy) by $\mathrm{t}$ test. The value of $\mathrm{P}<0.05$ was considered statistically significant.

\section{RESULTS}

The animals did not vary statistically with respect to age and body weight. Induction dose of the bolus combination of ketamine and xylazine ensured adequate anaesthesia and immobilization of all animals for tracheal intubation and epidural anaesthesia. Maintenance in the sternal position for 20 minutes, preparation of the surgical site and surgeries required additional anaesthesia in all of the dogs. The mean \pm SD duration of orchiectomy was $9 \pm 2$ minutes, while for the ovariohysterectomy was $16 \pm 4$ minutes. Statistically significant differences were not established between observed groups of animals in relation to the duration of surgical procedures. Evaluation of postoperative analgesia revealed low scores in all three groups of animals, throughout the complete 24-hours observation period. The appearance of vocalization or painful reaction to palpation of the wound surrounding tissue were absolutely absent. Values of 10 points, or higher, indicating the presence of moderate to severe pain and the necessity of additional analgesia, were not recorded. Comparison of the mean values of the postoperative pain evaluation between observed groups of animals revealed highest detected scores in L and LT groups four hours after EA, where the highest detected scores in group LM were recorded 12 hours after epidural (Fig. 1). Statistically significant differences were not established $(\mathrm{P}=0.654)$ between the observed groups comparing mean values of the postoperative pain assessment scores. Comparison of the mean values of pain assessment scores between males and females for the 24 hours postoperative period revealed significantly lower scores $(\mathrm{P}=0.04)$ in females. The average scores of postoperative pain assessment for males and females are presented in Fig. 2. The potential side effects of morphine application (pruritus at the site of the epidural application, constipation or urinary retention) were not detected among animals in the LM group.
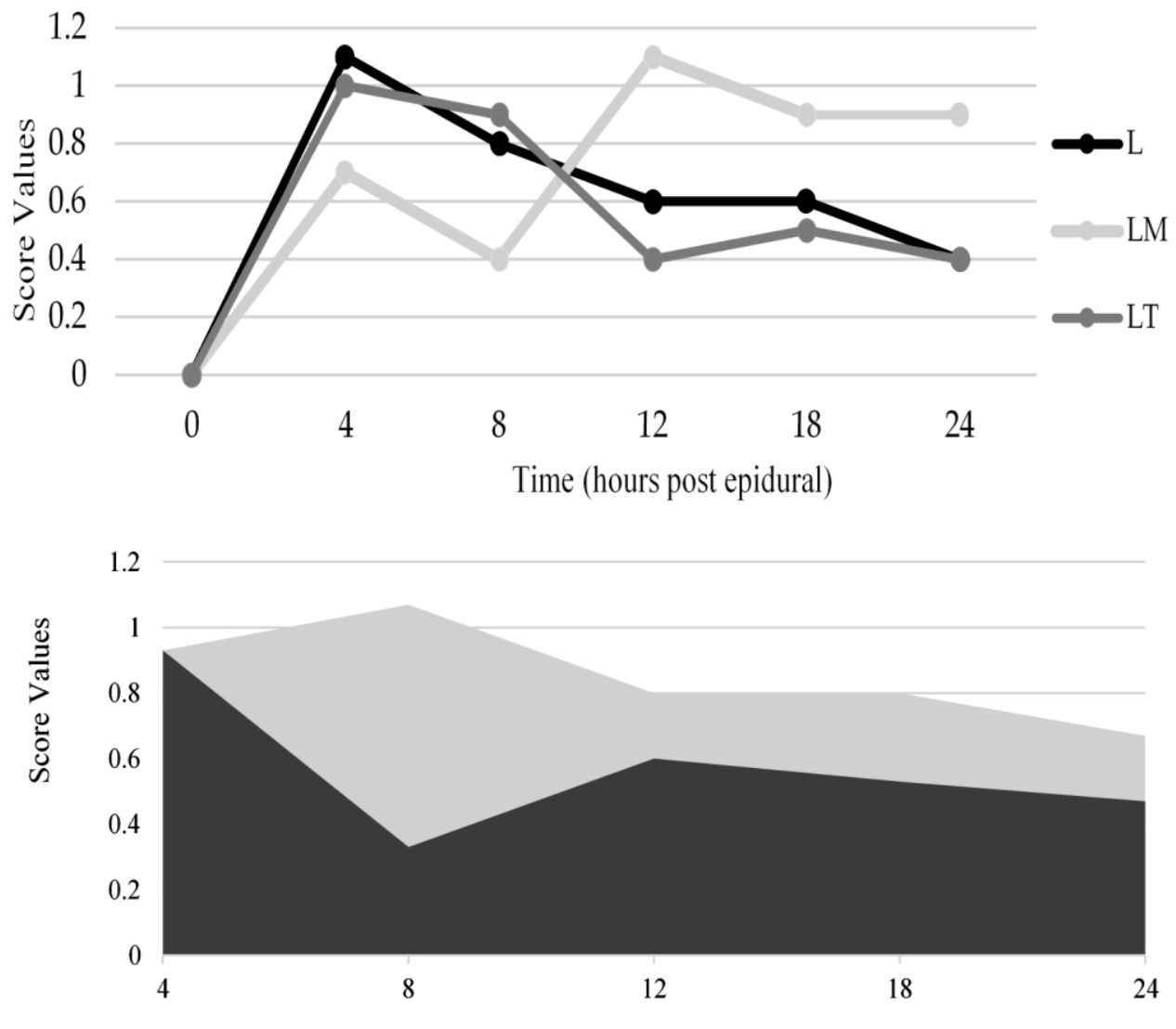

Time (hours post epidural)
Fig. 1: Average values of the pain assessment in group L, LM and LT during 24 hours of postepidural observation period.

Fig. 2: Average values of the pain assessment in males and females during 24 hours of postepidural observation period. 


\section{DISCUSSION}

Analgesic efficacy of single pre-emptive epidural administration of lidocaine alone, or in combination with morphine or tramadol, for the orchiectomy and initial 24 hours postoperative period has been recently described in dogs by Almeida et al. (2010). They concluded that epidural application of lidocaine with tramadol provides analgesic effect equivalent to application of lidocaine with morphine, at least during first 12 hours of postoperative observation period. Same as in our study, postoperative pain was evaluated using the University of Melbourne pain scale, while the used dose of lidocaine was higher $(6 \mathrm{mg} / \mathrm{kg}$ vs $4 \mathrm{mg} / \mathrm{kg})$. The highest pain score recorded in LM an LT group was 8 points. Similar, the results of our study indicate a highly potent analgesia after epidural administration of lidocaine with tramadol or morphine, but throughout complete 24 hours postoperative period of the observation. Detected values of postoperative pain evaluation were lower compared to the previous study. The highest pain score recorded in our study was 3 points. Furthermore, significant difference between our and the investigation of Almeida et al. (2010) was observed in relation to the efficiency of postoperative analgesia after epidural applications of lidocaine alone. In a previous study, application of lidocaine at a dose of $6 \mathrm{mg} / \mathrm{kg}$ did not provide adequate post-operative analgesia in most animals. Eleven of the 12 dogs required rescue analgesia during the first 12 hours of postoperative period. In our research, it was found that all the 12 dogs in group $\mathrm{L}$ experienced a similar level of postoperative analgesia to opioid (LT and LM) groups. Contradictory results could be explained by differences in the applied protocols of general anaesthesia. Authors of previous study (Almedia et al. 2010) induced general anaesthesia with thiopental, which is devoid of analgesic properties (Ngan Kee et al. 1997). On the other hand, induction of anaesthesia in the present investigation was carried out by intravenous application of a combination of ketamine and xylazine, both with a certain analgesic potential. Xylazine belongs to the group alpha- 2 adrenergic agonists, which are used for sedation or as an adjunct to intravenous or inhalatory general anaesthetics (Lemke 2004). Parenteral administration causes mild analgesia for up to 30 minutes (Rector et al. 1996). Ketamine is dissociative general anaesthetic with very potent analgesic effect. It has more analgesic mechanisms, of which primary one is antagonism at N-methyl-D-aspartate (NMDA) receptors. Secondary mechanisms of analgesia arise through interaction with $\mathrm{Ca}$ and $\mathrm{Na}$ channels, dopamine cholinergic receptors and inhibition of noradrenergic and serotonergic reuptake (Porter et al. 2015). Effect of the induction of general anaesthesia with ketamine on postoperative analgesia, as compared to induction with thiopental, was described in the research of Ngan Kee et al. (1997). In this study, patients undergone anaesthesia for caesarean section induced by ketamine, in contrast to the induction with thiopental, had significantly lower requirements for additional analgesia during 24 hours of postoperative recovery. Slingsby and Waterman-Pearson (2000) have shown that pre-emptive intramuscular administration of ketamine in dogs submitted for ovariohysterectomy significantly improved postoperative analgesia and reduced the need for additional analgesia. Furthermore, it is possible that the results of postoperative analgesia in our study reflects additive or synergistic effect between ketamine and used local anaesthetic and opioids. Synergistic or additive effects of concurrent application of ketamine and lidocaine in animals are visible from the studies investigating their effects on the minimum alveolar concentration of volatile anaesthetics (Barak et al. 2001; Wilson et al. 2008; Queiroz-Williams et al. 2016). Sen et al. (2005) have demonstrated that the combination of spinal administration of bupivacaine with intravenous ketamine provide more potent and longer lasting analgesia in comparison with bupivacaine alone. Similar effect reported Kararmaz et al. (2003), who combined intravenous ketamine and epidural bupivacaine and morphine. Analgesic synergism between ketamine and tramadol has been investigated in some previous studies. Webb et al. (2007) showed that pre-emptive intravenous administration of ketamine, in addition to tramadol analgesia, provides a significantly longer and more potent analgesia, compared to the application of tramadol alone. Analgesic benefit was evident over 48 hours of postoperative period, but it was most pronounced during the initial 24 hours. Further researches are needed in order to define the potential of analgesic synergism between ketamine, local anaesthetics and opioids in dogs.

In the present study, postoperative analgesic efficiency of used epidural protocols was also investigated after ovariohysterectomy. Detected values were even lower than those observed in animals underwent to orchiectomy. Authors of a recent systematic review of literature published during the last 40 years on postoperative analgesia with intravenous ketamine concluded that ketamine continuously exhibits potent analgesic effect, particularly pronounced after invasive surgeries, which is independent of the dose administered (Laskowski et al. 2011). Analgesic potency of different epidural protocols for ovariohysterectomy in dogs was investigated in few instances, with some inconsistent results. Pohl et al. (2012) compared quality of the epidural analgesia after administration of lidocaine alone or in combination with a different alpha- 2 adrenergic agonists. They concluded that the used epidural protocols were ineffective in ensuring adequate analgesia and anaesthesia for the ovariohysterectomy. As a potential reason they stated possibility of insufficient spreading of applied drugs to the area of the third and fourth lumbar nerve, from which origins innervation of the ligamentum ovaricum. Dose of lidocaine used in this study $(2.5 \mathrm{mg} / \mathrm{kg}$ of $1 \%$ lidocaine) was significantly lower comparing to our ( $4 \mathrm{mg} / \mathrm{kg}$ of $2 \%$ lidocaine), but the total volume was higher $(0.25 \mathrm{ml} / \mathrm{kg}$ compared to $0.2 \mathrm{ml} / \mathrm{kg})$. The significance of the dose of epidurally administered local anaesthetics on the efficiency of anaesthesia/analgesia is evident from the studies in human medicine (Liu et al. 1997; Sakura et al. 1998). Sakura et al. (1998) compared quality of sensory blockade after lumbar epidural application of $1 \%$ and $2 \%$ lidocaine, in an equal volume $(10 \mathrm{ml})$. Distribution over epidural space was identical for both applied concentrations of lidocaine, but the intensity of sensory blockade was significantly more pronounced after the administration of $2 \%$ lidocaine. Liu et al. (1997) described influence of the dose of local anaesthetic on the intensity 
of the sensory blockade and of its amount on the number of affected dermatomes. This study compared efficiency of $3 \%$ 2-chloroprocaine in an amount of $10 \mathrm{ml}$ over $1 \%$ solution of the same local anaesthetic in an amount of 30 $\mathrm{ml}$, resulting in an equivalent total dose of $300 \mathrm{mg}$ per bolus. Both of the solution provided the same intensity of sensory and motor blockade (same total dose), where $1 \%$ solution (greater total volume) affected significantly more dermatomes. Adequate efficiency of epidural lidocaine anaesthesia/analgesia for ovariohysterectomy in dogs was reported by Amnikov and Zlateva (2004). The dose of lidocaine was $6 \mathrm{mg} / \mathrm{kg}$, in an amount of $0.3 \mathrm{ml} / \mathrm{kg}$. Furthermore, for the same surgical procedure Almeida et al. (2007) have reached an effective epidural analgesia using bupivacaine $0.5 \%$ at a dose of $1 \mathrm{mg} / \mathrm{kg}(0.2 \mathrm{ml} / \mathrm{kg})$, while Neves et al. (2012) with application of epidural morphine alone $(0.1 \mathrm{mg} / \mathrm{kg})$ or tramadol $(2 \mathrm{mg} / \mathrm{kg})$, in dilution with sterile saline to a volume of $0.25 \mathrm{ml} / \mathrm{kg}$. In our study, established potency of postoperative analgesia following ovariohysterectomy, same as orchiectomy, reflects analgesic activity of drugs used for general (xylazine and ketamine) and epidural anaesthesia (lidocaine, morphine and tramadol), and their potential synergistic or additive effects.

\section{Conclusion}

In conclusion, this investigation demonstrated that compared epidural protocols provided efficient postoperative analgesia in all animals. Established quality of postoperative analgesia is not sole result of the epidurally applied drugs analgesic potency, but also analgesic activity of drugs used for general anaesthesia (xylazine and ketamine), and their mutual synergistic or additive effects. Further researches are needed in order to define the potential of analgesic synergism between different doses of ketamine and a most used local anaesthetics and opioids in dogs, with consideration of the used doses and route of their application.

\section{REFERENCES}

Acosta AD, Gomar C, Correa-Natalini C, Bopp S, Polydoro A and Sala-Blanch X, 2005. Analgesic effects of epidurally administered levogyral ketamine alone or in combination with morphine on intraoperative and postoperative pain in dogs undergoing ovariohysterectomy. American Journal of Veterinary Research 66: 54-61.

Almeida RM, Escobart A and Maguilnik S, 2010. Comparison of analgesia provided by lidocaine, lidocaine-morphine or lidocaine-tramadol delivered epidurally in dogs following orchiectomy. Veterinary Anaesthesia and Analgesia 37: 542-549. https://doi.org/10.1111/j.1467-2995. 2010.00563.x.

Almeida TF, Fantoni DT, Mastrocinque S, Tatarunas AC and Imagawa VH, 2007. Epidural anesthesia with bupivacaine, bupivacaine and fentanyl, or bupivacaine and sufentanil during intravenous administration of propofol for ovariohysterectomy in dogs. Journal of American Veterinary Medical Association 230: 45-51.

Amnikov B and Zlateva N, 2004. Epidural anaesthesia for castration of the bitch. Bulgarian Journal of Veterinary Medicine 7: 113-119.

Barak M, Ben-Shlomo I and Katz Y, 2001. Changes in effective and lethal dose of intravenous anesthetics and lidocaine when used in combination in mice. Journal of Basic and Clinical Physiology and Pharmacology 12: 315-322.
Desborough JP, 2000. The stress response to trauma and surgery. British Journal of Anaesthesia 85: 109-117.

Firth AM and Haldane SL, 1999. Development of a scale to evaluate postoperative pain in dogs. Journal of American Veterinary Medical Association 214: 651-659.

Guedes AGP, Natalini CC, Robinson EP, Alves SDL and Oliveira ST, 2005. Epidural administration of tramadol as an analgesic technique in dogs submitted to stifle surgery. International Journal of Applied Research in Veterinary Medicine 3: 351-359.

Gusak V, Turkovic V, Nesek-Adam V, Lerotic I, Popovic M, Brajenovic N, Karaconji IB and Vnuk D, 2013. Lidocaine Serum concentration after epidural administration in combination with morphine and fentanyl in rabbit - a preliminary study. Research in Veterinary Science 94: 651655. https://doi.org/10.1016/j.rvsc.2012.10.001

Habibian S, Bigham AS and Aali E, 2011. Comparison of lidocaine, tramadol, and lidocaine-tramadol for epidural analgesia in lambs. Research in Veterinary Science 91: 434438. https://doi.org/10.1016/j.rvsc.2010.09.023

Halder S and Bose PK, 2000. Post-operative analgesic effect of epidural xylazine in combination with tramadol in dog. Indian Journal of Animal Health 39: 51-52.

Hendrix PK, Raffe MR, Robinson EP, Felice LJ and Randall DA, 1996. Epidural administration of bupivacaine, morphine or their combination for postoperative analgesia in dogs. Journal of American Veterinary Medical Association 209: 598-607.

Hodgson PS, Liu SS and Gras TW, 1999. Does epidural anesthesia have general anesthetic effects? A prospective, randomized, double-blind, placebo-controlled trial. Anesthesiology 91: 1687-1692.

Holton L, Reid J, Scott EM, Pawson P and Nolan A, 2001. Development of a behaviour-based scale to measure acute pain in dogs. Veterinary Record 148: 525-531. https://doi.org/10.1136/vr.148.17.525.

Jones RS, 2001. Epidural analgesia in the dog and cat. Veterinary Journal 161: 123-131.

Kararmaz A, Kaya S, Karaman H, Turhanoglu S and Ozyilmaz MA, 2003. Intraoperative intravenous ketamine in combination with epidural analgesia: postoperative analgesia after renal surgery. Anaesthesia and Analgesia 97: 1092-1096.

Laskowski K, Stirling A, McKay WP and Lim HJ, 2011. A systematic review of intravenous ketamine for postoperative analgesia. Canadian Journal of Anesthesia 58: 911923. https://doi.org/10.1007/s12630-011-9560-0.

Lemke KA, 2004. Perioperative use of selective alpha-2 agonists and antagonists in small animals. Canadian Journal of Anesthesia 45: 475-480.

Liu SS, Ware PD and Rajendran S, 1997. Effects of concentration and volume of 2-chloroprocaine on epidural anesthesia in volunteers. Anesthesiology 86: 1288-1293.

Mastrocinque S, Almeida TF, Tatarunas AC, Imagawa VH, Otsuki DA, Matera JM and Fantoni DT, 2012. Comparison of epidural and systemic tramadol for analgesia following ovariohysterectomy. Journal of American Veterinary Medical Association 48: 310-319. https://doi.org/10.5326/ JAAHA-MS-5795.

Moll X, Frenso L, García F, Prandi D and Andaluz A, 2011. Comparison of subcutaneous and transdermal administration of buprenorphine for pre-emptive analgesia in dogs undergoing elective ovariohy-sterectomy. Veterinary Journal 187: 124-128. https://doi.org/10.1016/ j.tvj1.2009.11.011.

Morton CM, Reid J and Scott EM, 2005. application of a scaling model to establish and validate an interval level pain scale for assessment of acute pain in dogs. American Journal of Veterinary Research 66: 2154-2166. 
Mwangi WE, 2020. Epidural lidocaine and medetomidineketamine anaesthesia in feline orthopedic surgery. International Journal of Veterinary Science 9: 164-167.

Neves CS, Balan JAO, Pereira DR, Stevanin H and Cassu RN, 2012. A comparison of extradural tramadol and extradural morphine for postoperative analgesia in female dogs undergoing ovariohysterectomy. Acta Cirurgica Brasileira 27: 312-317.

Ngan Kee WD, KS Khaw, Ma ML, Mainland PA and Gin T, 1997. Postoperative analgesic requirement after cesarean section: a comparison of anesthetic induction with ketamine or thiopental. Anaesthesia and Analgesia 85: 1294-1298.

Pekcan Z and Koc B, 2010. The post-operative analgesic effects of epidurally administered morphine and transdermal fentanyl patch after ovariohysterectomy in dogs. Veterinary Anaesthesia and Analgesia 37: 557-565.

Pohl VH, Carregaro AB, Lopes C, Gehrcke MI, Muller DCM and Garlet CD, 2012. Epidural anesthesia and postoperatory analgesia with alpha-2 adrenergic agonists and lidocaine for ovariohysterectomy in bitches. Canadian Journal of Veterinary Research 76: 215-220.

Porter SB, McClain RL, How BL, Ardon AE, Mazer LS, Knestrick BM and Clendenen AM, 2015. Perioperative ketamine for acute postoperative analgesia: The Mayo Clinic-Florida experience. Journal of Perianesthesia Nursing 30: 189-195.

Qayyum A, Hussain R, Ali F and Ghori MT, 2020. Surgical intervention of hypospadias associated with urethral diverticulum in a male goat kid: A case report. Agrobiological Records Volume 1: 31-33. https://doi.org/ 10.47278/journal.abr/2020.006

Queiroz-Williams P, Doherty TJ, da Cunha AF and Leonardi C, 2016. Effects of ketamine and lidocaine in combination on the sevoflurane minimum alveolar concentration in alpacas. Canadian Journal of Veterinary Research 80: 141-145.

Rauser P, Lexmaulova L, Vlasin M, Fichtel T and Lorenzova J, 2004. Effect of epidural administration of lidocaine, fentanyl and their combination on the minimum alveolar concentration of halothane in dogs. Veterinary Medicine Czech 49: 421-426.

Rector E, Otto K, Kietzmann M, Nolte I and Lehmacher W, 1996. Pharmacokinetics and Effects of xylazine (rompun) in dogs. Berliner und Münchener tierärztliche Wochenschrift 109: 18-22.
Sakura S, Sumi M, Yamada Y, Saito Y and Kosaka Y, 1998. Quantitative and selective assessment of sensory block during lumbar epidural anaesthesia with $1 \%$ or $2 \%$ lidocaine. British Journal of Anaesthesia 81: 718-722.

Sen S, Ozmert G, Aydin ON, Baran N and Caliskan E, 2005. The persisting analgesic effect of low-dose intravenous ketamine after spinal anaesthesia for cesarean section. European Journal of Anaesthesiology 22: 518-523.

Slingsby LS and Waterman-Pearson AE, 2000. The PostOperative Analgesic Effects of ketamine after canine ovariohysterectomy - a comparison between pre- or postoperative administration. Research Veterinary Science 69: 147-152.

Torske KE and Dyson DH, 2000. Epidural analgesia and anesthesia. Veterinary Clinics of North America: Small Animal Practice 30: 859-874.

Tusell JM, Andaluz A, Prandi D, Costa C and García F, 2005. Effects of epidural anaesthesia-analgesia on intravenous anaesthesia with propofol. Veterinary Journal 169: 108112.

Valverde A, 2008. Epidural analgesia and anesthesia in dogs and cats. Veterinary Clinics of North America: Small Animal Practice 38: 1205-1230.

Vettorato E, Zonca A, Isola M, Villa R, Gallo M, Ravasio G, Beccaglia M, Montesissa C and Cagnardi P, 2010. Pharmacokinetics and efficacy of intravenous and extradural tramadol in dogs. Veterinary Journal 183: 310-315. https://doi.org/10.1016/j.tvj1.2008.11.002

Webb AR, Skinner BS, Leong S, Kolawole H, Crofts T, Taverner M and Burn SJ, 2007. The addition of a smalldose ketamine infusion to tramadol for postoperative analgesia: a double-blinded, placebo-controlled, randomized trial after abdominal surgery. Anaesthesia and Analgesia 104: 912-917.

Wetmore LA and Glowaski MM, 2000. Epidural Analgesia in Veterinary Critical Care. Clinical Techniques in Small Animal Practice 15: 177-188. https://doi.org/10.1053/ svms.2000.16545

Wilson J, Doherty TJ, Egger CM, Fidler A, Cox S and Rohrbach B, 2008. Effects of intravenous lidocaine, ketamine, and the combination on the minimum alveolar concentration of sevoflurane in dogs. Veterinary Anaesthesia and Analgesia 35: 289-296. https://doi.org/10.1111/j.1467-2995.2007. 00389.x 